\title{
VII.
}

Aus der Kgl. Universitäts-Ohrenklinik zu Halle a. S.

Direktor Geh.-R. Prof. Dr. H. Sehwartze.

\section{Nasale Auskultation des Ohres bei Katheterismus tubae.}

\author{
Von \\ Dr. Laval, Hilfsassistent der Klinik.
}

(Mit 3 Abbildungen.)

Die Ansichten tiber den diagnostischen Wert der Untersuchungsergebnisse bei dem Katheterismus tubae gehen bekanntlich noch weit auseinander. Zum Teil werden sie übersehätzt, mehr jedoch wohl mit Unrecht untersehätzt. Die Gründe dafür sind unzweifelhaft in dem anßerordentlich komplizierten Bau des Tractus auditorius, dem Entstehungsorte der Sehallphänomene und in den tuberaus vielgestaltigen und abwechslungsreichen anatomisehen Veränderungen bei Krankheitsprozessen zu suchen. Diese Faktoren bedingen naturgemäb Differenzen in Eigenart und Stärke der Auskultationserseheinungen, Differenzen, die nicht etwa stets dentlich und seharf begrenzt, sondern oft so gering sind, daß die Phänomene ineinander überzugehen scheinen.i Dem subjektiven Urteil ist dadurch ein breiter Spielraum gelassen.

Besonders ist es nach dem üblichen Auskultationsverfahren zuweilen unmöglieh, den Ursprungsort der Geräusche festzustellen. Schon Deleau besehrieb allerdings das „bruit de pavillon", das - im Rachen entstehend und mit dem unbewaffneten Ohre wahrnehmbar - wohl zu trennen sei von den vom Ohre ans wahrnehmbaren Phänomenen. An unserer Klinik wenden wir das Verfahren an, den Auskultationssehlaueh abwechselnd $z \mathfrak{n}$ sebließen und zu öfnen, und unterseheiden so im Pharynx und im Ohr entstehende Geräusche. Zur Differenzialdiagnose zwischen Tuben- und Paukengeräuschen richteten wir uns nach der Schallstärke: laute Geräusche verlegten wir in die Pauke und nannten sie nahe, für leisere, entfernte Geräusche nahmen 
wir die Tube als Entstehungsort an. Doeh schien mir dies nicht für alle zwischen Kathetermündung und Trommelfell entstehenden Geräusche sichere Unterscheidungsmerkmale zu geben. Politzer giebt an, daB die meisten Rasselgeräusche in der Tube entständen, belegt jedoch die Ansicht meines Wissens nicht dureh Versuche. Ferner hat Uffen orde in seinen Experimenten dargetan, dab in der Tube entstehende Geräusche genau so laut und "nah" durch den Auskultationssehlauch gehört werden, als die in der Panke erzengten, wenn von der Tube her eine ununterbrochene Luftsäule bis zum Trommelfell reicht. Es ist anch zu bedenken, daß laute und leise Geräusehe, beide von differentem Charakter, sowohl in der Pauke als anch in der Tube entstehen können. Es ist somit die Möglichkeit gegeben, bei lokalisiertem Tabenkatarrh, bei freier Paukenhöhle, aus Rasselgeräuschen, die in der katarrhalisch erkrankten Tube entstehen, fälsehlich die Diagnose auf Exsudat in der Pauke zu stellen. Ähnliche Irrtümer können aus dem einseitigen, durch das $0 \mathrm{hr}$ allein aufgenommenen Auskultationsbefunde auch sonst gezogen werden.

Ich wurde dadureh in diesen Gedankengang gedrängt, daß durch die von Grunert angeregten Untersuchungen Uffenordes ${ }^{1}$ ) an unserer Klinik die ganze Bewertung der Auskultationsphänomene in Flub gekommen war und dieser Untersuchungsmethode eine intensivere Beachtung geschenkt wurde.

Ich entschlob mich, die Geränsche, die ich durch den Anskultationsschlauch wahrnehmen konnte, durch Auskultation von der Nase aus zu kontrollieren. Nachdem ich so bereits einige positive Resultate erhalten hatte, prüfte ich das Verfahren an mehreren Leichen nach. Diese zahlreichen Versuche fielen völlig ubereinstimmend mit den am Lebenden gewonnenen Erfahrungen aus und bildeten die Grundlage für weitere klinische Untersuchungen.

Bei späterem Nachlesen in der Literatur fand ich, daß dieser Weg - äußerer Gehörgang bis zum Naseneingang - wiederholt zu Experimenten benutzt ist. Ich erinnere an Politzers Stimmgabelversuch zur Untersuchung der Tube auf ihre Wegsamkeit. ${ }^{2}$ ) Derselbe Autor hat empfohlen, bei Versagen des Katheterismus und seiner Ersatzverfahren, zu derselben Untersuchung den um-

1) Uffenorde: Beiträge zur Auskultation der Mittelohrräume, Arch. $f$. Ohrenheilk., LXXI. Bd., S. 1-30.

2) Wiener medizinische Wochenschrift 1892. 
gekehrten Weg einzuschlagen. Er verdichtet die Luft im äußeren Gehörgang und auskultiert dabei die Tube durch einen luftdicht in die Nase eingeführten Hörsehlanch. ${ }^{1)}$ Von diesen Verfahren unterscheidet sicb jedoch das meinige, abgesehen von der Technik, wesentlich. Denn es kann bei jeder Luftdusehe also in weit größerem Umfange and bequem angewandt werden und ist geeignet, bei Zweifeln über -den Ursprungsort von Geräuschen, diesen mit bisher unbekannter Sicherheit erkennen zu lassen. Es ermöglicht daher eine genanere Beurteilung pathologiseher Veränderungen und erschliebt einen klaren Einblick in gewisse mit dem Katheterismus tubae auftretende Erscheinungen. Das höchst einfache und bei einiger Intelligenz des Patienten leicht mit seiner Unterstützung ausführbare Verfahren ist folgendermaßen.

$\mathrm{Zu}$ meinen Versuchen verwandte ich ausschlieblich das an unserer-Klinik in Gebrauch befindliche L u c a e sche Doppelgebläse, versehen mit der $\mathrm{Z}$ a u fal sehen Desinfektionskapsel, an, das mit Hilfe eines Gänsefederkiels mit dem trichterförmigen äußeren Ende des Katheters verbunden wurde. Tch benutzte, um Differenzen von seiten des Katheters auszuschließen, nur mittelweite Instrumente mit olivenförmigem Schnabelende. Die Auskultationsschläuche waren $70-80 \mathrm{~cm}$ lang, hatten $8 \mathrm{~mm}$ lichte Weite und keine Ansatzstücke. Die für Arzt und Patienten bestimmten Fnden sind dadurch gekennzeichnet, dah erstere sehräg, letztere quer abgeschnitten sind.

Wenn ich nach Einführung des Katheters das zu untersuchende $\mathrm{Ohr}$ in üblicher Weise mit meinem gegenuberliegenden Ohre verbunden hatte, verband ich mein anderes Ohr mit dem entgegengesetzten, nicht durch den Katheter versperrten Naseneingang des Patienten so, daB das Schlauchende etwa $2-3 \mathrm{~cm}$ weit schräg nach oben hinten bis in den Anfangsteil des mittleren Nasengangs vorgeschoben wurde. Der Schlauch wurde hier durch gelinden Fingerdruck auf den Nasenflügel locker fixiert. Ich ließ den Patienten durch den offenen Mund atmen und verbot ihm das Sprechen, solange der Schlauch in der Nase steckte. Denn die Sprachlaute dringen so laut und schmerzhaft wie Donnerschläge ans $\mathrm{Ohr}$. Teh gab nun dem Patienten auf, je nach meiner Aufforderung "links" bzw. "rechts" bald den linken, bald den rechten Schlauch durch Fingerdruck fest zu scbließen. Zunächst prüfte ich nun bei ganz gelindem Luft-

1) Annales des maladies de l'Oreille XVIII. 3. p. 206.1892 , 
druck den Tubeneingang anf etwaiges Vorhandensein von Sekret, bald vom Ohre aus horchend, ob der Luftstrom nicht etwa zu stark sei und von hier nabe gehört würde, bald die Tube von der Nase auskultierend. In den meisten Fällen gelingt es, durch einige vorsichtige Lufteintreibungen die Tube von Sekret so weit zu süubern, daß die Rasselgeräusche verschwinden. Dann trieb ich einen stärkeren Luftstrom bis in die Paukenhöhle, indem ich den "Nasenschlauch" sehließen lieB. Von da ab lieb ich, entsprechend den einzelnen Lufteintreibungen, die Auskultationsschläuche abwechselnd schließen und öffnen.

In einzelnen Fällen führte ich aus äußeren Gründen den Hörsehlauch abwechselnd ins Ohr und in die Nase, doch kam ich wenigstens am Lebenden aus ästhetischen Gründen bald wieder davon ab.

\section{Versuche an der Leiche.}

Anders an der Leiche. Hier verwandte ich nur einen Hörschlauch, den ich nach Bedarf umwechselte, und verstopfte mir bei den meisten Versuchen das unbewaffnete Ohr mit erweichtem Paraffin. Ioh vermied so die Wabrnehmung störender Nebengeräusche.

Die Versuche, die ich nur an Leichen Erwachsener versehiedenen Lebensalters, meistens von mittlerem, je einmal von kräftigem und stark reduziertem Ernährungszustande vornahm, sind folgende. Die Sektion sämtlicher Körperhöhlen war in allen Fällen vorher ausgeführt, nur die Halsorgane waren in situ gelassen worden.

I. Ich ließ bei zwei Leichen nach Einführung des Katheters, nachdem ich mich von der Wegsamkeit und Sekretfreiheit der Tube überzeugt hatte, eine ziemlich dickflüssige Masse, Glyzerin, den unteren Nasengang herablaufen. Der Kopf der Leiche lag dabei horizontal in der Verlängerung des Körpers und etwa um 450 nach der zu untersuchenden Seite gedreht. Sehon bei der ersten Lufteintreibung hörte ich auch mit unbewaffnetem Ohre laut brodelnde und flatternde Geränsche - bruit de pavillon -, unzweifelhaft entstanden dureh die vom Luftstrom aufgeworfenen Blasen und Wellen des Glyzerins, deren Schwingungen sich einige Male sogar durch vibrierende Bewegung des Katheters fiublbar machten. Bei Auskultation des Ohres hörte ich ein zusammengesetztes Geräusch, in dem das Blasen zwar deutlich zu erkennen, aber durch dumpf und fern klingende Rasselgeräusche 
von sonorem, unregelmäßigem Charakter teilweise verdeckt war. Von der Nase aus hörte man das typische bruit de pavillon naturgemäß in sebr großer Nähe und intensiver Deutlichkeit.

II. Nach völliger Reinigung beider Tubenostien dureh etwa etwa zehnmaliges Ausblasen mit dem Katheter bei mäßiger Hochlagernng des Kopfes eröffnete ich bei einer dieser Leichen die rechte Tube von der durch die Sektion bereits freigelegten Schädelbasis aus, und zwar an der Grenze des knöchernen und knorpeligen Abschnitts. Ich lieh nun durch eine Hilfsperson innerhalb des freigelegten Tubenlumens mit einer metallischen Sonde scharrende und knipsende Geräusche ausführen. Leicht konnte ich mich taberzengen, dal diese Geräusche von $\mathrm{Obr}$ und Nase der Leiche gleich deutlich hörbar waren. Dasselbe Resultat erzielte ich auf der linken Seite bei einem ähnliehen Versuch.

Durch Wegmeißeln der zwisehen vorderem Tubenteil und Foramen ovale liegenden Knochenpartie legte ich die obere und vordere Wand des knorpeligen Tubenabschnitts in seinem hinteren Teile frei. Ich konnte jetzt durch Anpressen der hinteren Wand an die vordere und sehnelles Wiederloslassen mehrfach ein tickendes, knackendes Geräusch hervorrufen (vgl. Versuch Uffenordes), das ebenfalls von Ohr und Nase aus hörbar war.

III. Bei diesen Experimenten lagerte ich den Kopf der Leiche durch Unterstiutzung und leichte Drehung nach der Seite so, daß die Achse der Tube fast horizontal, ganz wenig nach hinten geneigt verlief. Ich erzielte dadurch, dab Flitssigkeit nur schwer aus der Pauke über die Antrumschwelle in den Warzenfortsatz laufen konnte, und verhinderte mit großer Sicherheit ein Eindringen in die Tube, so daß diese dauernd frei von Flüssigkeit blieb.

Trommelfell leicht getrübt, sonst ohne Besonderheiten. Auskultation des Ohres ergibt bei leicht ausführbarem Katheterismus leichtes zähes Rasseln in der Tube, das von Ohr und Nase aus, hier etwas deutlicher, hörbar ist. Nach etwa zehn Lufteintreibungen ist die Tabe völlig frei, man hört jetzt weiches normales Blasegeräusch, lauter von der Nase als vom Ohre aus, doeh von gleichem Charakter. Eröffnung des Aditusdaches, Entfernung des Amboh'. Auskultation ergiebt bei geschlossener künstlicher Öffnung denselben Befund wie zuvor, es sind daher keine Nebenverletzungen, besonders des Trommelfells, eingetreten. Ausfüllung der ganzen Pauke, des Antrum nebst Zellen des Warzen- 
fortsatzes mit Wasser mittels einer Pravazschen Spritze. Luftdusche bei ganz leichtem Druck ergibt bei Auskultation durch die Nase Blasegeräusch, die Tube ist demnach frei. Vom Obre aus ist das Blasegeräusch leise (entfernt hörbar), die Luft tritt demnach nicht in die Pauke ein. Einführung einer dünnen Silberkanüle durch das eröffnete Aditusdach bis auf den Boden der Paukenhöhle. Treibt man nun unter gelindem Druck Luft durch die Kanüle, so treten zahlreiche Luftbläschen aus, die die Flïssigkeit durehdringend im Aditus auftauchen und platzen. Dementsprechend hört man auch auffallend laut und nahe prasselnde und rasselnde Geräusche bei Auskultation durch das Ohr. Die Flïssigkeit wurde durch Verlust mitgerissener Wasserteileben allmählich geringer - nicht dureh $A b f l a b$ in die Tube, die dureh zeitweilig eingesehaltete Luftdusche mit gleichzeitiger Auskultation dureh die Nase als danernd frei festgestellt wurde - , so daß das Rasseln zuletzt ganz feinblasig und leiser, jedoch immer noch mindestens ebenso laut wie beim Lebenden wurde. Schlieblich war nur nooh ein Blasegeräuseh, vermischt mit deutlichem hohen Pfeifen, wahrnehmbar, das sicherlich durch bängende in Vibration versetzte Wasserteilchen erzeugt wurde.

Bei Auskultation duroh die Nase, die ich alternierend mit der durch das $\mathrm{Ohr}$ vornahm, wobei mein eines $\mathrm{Ohr}$ mit dem Hörsehlauch bewaffnet, das andere mit der Hand bedeckt war, glaubte ich nur einmal ein ganz außerordentlich leises und feines Rasseln zu hören. Ich verstopfte mir deshalb zur Nachpriffung das unbewaffnete $\mathrm{Ohr}$ mit erweichtem Paraffin. Jetzt waren die Geräusche völlig und dauernd verschwunden, obwohl ich das Experiment mindestens $5-6$ mal wiederholte Es ist daher gewiß, daß ich die von der eröffneten Schädelbasis her lant wahrnehmbaren Rasselgeräusche mit dem ungenügend verschlossenen $\mathrm{Ohr}$ perzipiert hatte. Das Fortbestehen der Rasselgeräusche wurde außerdem dureh die Hilfsperson kontrolliert, die die Luft durch die Kanüle in die Paukenhöhle eintrieb.

Man könnte vielleicht annehmen, daß die Tube kollabiert war, so daß keine kontinuierliehe Luftsäule vorhanden war, der den Schall von der Pauke nach dem Epipharynx fortpflanzen konnte. Doch fand ich keinen Unterschied, ganz gleich, ob ich keinen Katheter eingefuhrt hatte, oder das Instrument in der Tube lag, ob ich durch Zug nach vorn und Andrücken an das Septum das Tubenostium zum Klaffen brachte, oder ob ich gar 
dureh vorsichtig ausgeführte Luftdusehe die Tnbenwände auseinanderdrängte. Im letzteren Falle hörte ich nur ein weiches gleichmäBiges Blasegeräuseh. Tob habe auch keinen Untersehied finden können, wenn ich eben erst die Paukenhöhle mit Wasser frisch gefüllt hatte, oder wenn nur noch wenig Wasser darin vorhanden war. Es fällt damit, zumal wenn man die Kopfhaltung berücksichtigt, auch der Einwand, daß etwa eine Wassersäule zentralwärts vom Isthmus tubae ein Hindernis für die Schalleitung abgegeben hätte.

Links fand sich bei dieser Leiche ein normales Trommelfell, der Katheterismus tubae ergab ein normales weiches Blasegeräusch. Das Ergebnis meiner Versuche auf dieser Seite deckt sich völlig mit dem soeben beschriebenen. Ein Zweifel konnte mir hier um so weniger unterlaufen, als ich von nun ab stets mein unbewaffnetes $\mathrm{Ohr}$ mit Paraffin verstopfte.

Diesen Versuch, mit dem ich feststellen konnte, daß selbst unverhältnismäßig laute, lediglich in der Pauke entstehende Geräusche nicht von der Nase aus wahrnehmbar sind, habe ich noch beiderseits an zwei weiteren Leichen, also im ganzen an sechs Schläfenbeinen in sita geprüft, eine Zahl, die meines Ermessens wohl genügt, um die allgemeine Gültigkeit dieses Befundes darzutun und eine etwaige gegenteilige Erfahrung als Ausnahme erscheinen zu lassen.

IV. An zwei weiteren Leichen führte ich folgenden Versuch aus, ich erprobte ihn also an vier Schläfenbeinen in situ. Bemerken muß ich noch, daß ich bei der ersten von diesen Leichen rechts im Beginn des normalen Blasegeräusches ein typisches (Tip) Anschlagegeränsch konstatierte. Dieses war von der Nase aus nicht hörbar.

Ich hatte bereits vorher bei einigen Leichen 4-5 Tropfen Wasser durch den Katheter mittels vorsichtiger Luftdusche in die Ohrtrompete eingetrieben und so Rasselgeräusche erzielt, die von $\mathrm{Ohr}$ und Nase aus gleich laut hörbar waren; doch vermißte ich ein diagnostisches Merkmal für eine exakte Bestimmung des Entstehungsortes jener Geräusche.

Ich eröffnete deshalb die Tube von oben, d. h. von der freiliegenden Schädelbasis her durch partielles Wegmeißeln ihres knöchernen Daches dicht hinter dem Foramen spinosum. Ich legte so das Tubenlumen etwas nach vorn vom Isthmus, dicht vor der Grenze des knorpeligen und knöehernen Abschnitts, auf etwa $2 \mathrm{~mm}$ frei. Der Kopf lag bei dieser Versuchsreihe so, dab die Tube fast 
horizontal, jedoch mit dem Ostium pharyngeum etwas tiefer lag. Während durch meine Hilfsperson von oben langsam tropfenweise Wasser in die Öfnung geschickt wurde, trieb ich mit der Luftdusche einen Luftstrom in die Tube. Es entstand im Gegensatz zu den in der Pauke erzengten wesentlich leisere Geräusch, klingendes Rasseln und Platzen von Blasen. Diese Geräusche waren gleich stark von der Nase und vom Ohr aus hörbar. Der Lnftstrom preßte sich größtenteils durch die artifizielle Öffnung des Tubendaches hindurch, da die springenden Blasen und die Wassertröpfeben diesen Weg nahmen. Von Zeit zu Zeit reinigte ich die Tube durch eine kräftige Luftdusche bei freier und bei geschlossener Öffnung im Tubendach und uberzeugte mich, daß auch jetzt keine Geräusche von der Pauke aus hörbar waren, sobald die Tube leer war.

Wenn ich nun die Resultate dieser Versuche kritisch znsammenfasse, so muB ich zunächst anerkennen, daß, abgesehen vom ersten Experiment, es mir nicht gelungen ist, die natürlichen Verbältnisse exakt nachzuahmen. Ich glaube jedoch, daß ich jeden Versuch, diesem Ideale näher zu kommen, mit einer weit geringerèn Möglichkeit der Kontrolle und einem ähnlichen Maße der Unsicherheit hätte bezahlen müssen, mit der wir der Beurteilung der Auskultationsphänomene am Lebenden hinsichtlich ihrer Lokalisation gegentiberstehen. Dagegen ermangelt es den Versuchen nicht an Vorzügen insofern, als unsere Resultate, weil unter ersohwerenden Umständen gewonnen, am Lebenden einen um so sichereren Wert erlangen. Denn die Rasselgeräusche, die wir nach Eröffnung des Aditusdaches in der Paukenhöhle erzeugten, sind sicher ganz erheblich stärker und lauter, als unter normalen Umständen. Hier hatten wir auch keine mit den Geräuschen zeitlich zusammenfallenden Luftströmungen in der Tube, die sioh der Sehalleitnng nach dem Epipharynx erschwerend in den Weg stellen konnten. Um wieviel weniger werden wir am Patienten diese Geräusche per nares hören können, wenn diese Luftbewegungen vorhanden sind, und das starke Blasegeräusch am Tubenostium hinzutritt. Dieser Luftstrom ist es auch, der bei Versuch IV der Auskultation durch die Nase stärker hindernd entgegentritt, als beim Lebenden, wo die Luff in den meisten Fällen, d. h. bei intaktem Trommelfell, nicht durch eine Gegenöffnung entweichen kann. Können wir also unsere Erfahrungen an der Leiche aus diesen Gründen um so sicherer auf die klinischen Untersuchungen über- 
tragen, so lege ich das Hauptgewicht auf die mit Sicherheit gelungene Lokalisation der Geräuschentstehung und die Wahrnehmbarkeit dieser Schallerscheinungen von Ohr und Nase aus. Das Ergebnis ist demnach folgendes:

1. Geräusche, die am Tubenostium entstehen, sind sehr laut durch die Nase, häufig auch obne Zuhilfenahme des Hörschlauches, dagegen dumpf, undeutlich, verschwommen und entfernt klingend, zuweilen nur als sonorer Beiklang vom Ohre aus wahrnehmbar.

2. Geräusche, die in der Tube entstehen, sind von Ohr und Nase aus hörbar, und zwar etwa gleich stark.

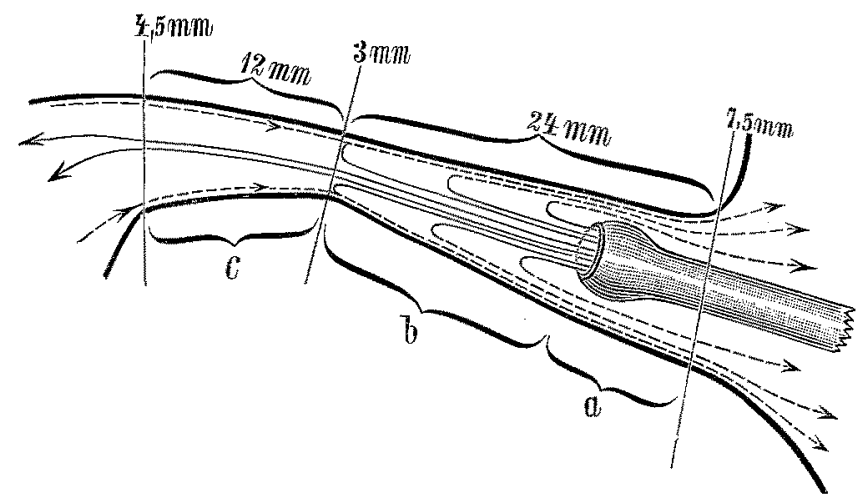

Fig. I.

3. Geräusche die in der Pauke ihren Ursprung nehmen, sind nur vom Ohre, nicht ron der Nase aus hörbar.

Die Luftströmungen beim Katheterismus tubae.

Ich habe versucht, mir an der Hand des anatomischen Präparates diese Phänomene physikaliseh zu erklären und bin dabei zu folgenden Vorstellungen gelangt, die wohl durch beistehende Skizze anschanlicher gemacht werden. Die Länge der ganzen Tube nehme ich auf $36 \mathrm{~mm}$, des knöchernen Abschnitts auf $12 \mathrm{~mm}$ und des knorpelig-membranösen auf $24 \mathrm{~mm}$ an. Die lichte Höhe des Kanals beträgt am Ostium tympanicum $4,5 \mathrm{~mm}$, am Isthmus $3 \mathrm{~mm}$, am Ostium pharyngeum $7,5 \mathrm{~mm}$. Die lichte Weite in horizontaler Ausdehnung $3,3 \mathrm{~mm}, 1 \mathrm{~mm}$ und $5 \mathrm{~mm}$. Ich nehme an, daß der Schnabel des Katheters etwa $8 \mathrm{~mm}$, also bis zur 
Grenze des äußeren und mittleren Drittels des knorpeligen Tubenabschnitts vordringt. In diesem annähernd sandubrförmigen Kanal und den Mittelohrräumen strömt bei der Luftdusche die Luft aus dem Katheterschnabel zunächst breit in die Pauke und ihre Adnexe ein, bis sich der Luftdruck hier mit dem im hinteren knorpeligen Tubenabschnitt (zwisehen Kathetersehnabel und Isthmus tubae) ausgeglichen hat. Wirkt nun von jetzt ab ein praktiseh betrachtet gleichmäßiger Luftstrom, wie wir ihn mit dem Lucäschen Doppelgebläse erzielen können, weiter ein, so werden sich bestimmte Luftströmungen bilden müssen, bis schließlich bei Aufhören des Überdruckes die komprimierte Luft ans der Pauke entweicht.

Unterscheiden wir zunächst drei Tubenabschnitte,

a) Ostium pbaryngeum bis Katheterschnabel,

b) Katheterschnabel bis Isthmus tubae,

c) Isthmus tubae bis Ostium tympanicum,

so ist die Luftbewegung im Abschnitt a) am einfaehsten, denn hier findet sich aussehlieflich ein energischer Luftstrom in der Richtung nach dem Rachen zu. Die Luftbewegung hier ist aber auch die kräftigste im ganzen System der Mittelohräume, weil alle Luft, die den Katheter passiert, hier in einheitlichem Strome wieder entweichen mub. Deshalb erzengen wir auch hier abgesehen von den zuweilen sehr lauten hohen, pfeifenden Perforationsgeräuschen - bei weitem die stärksten Geränsche, die häufig schon in einiger Entfernung deutlich wabrnehmbar sind. Daneben begegnen wir hier auch mittelstarken und leisen Geräuschen, von denen die letzteren nur durch den in die Nase eingeführten Auskultationssehlanch perzipiert werden können.

Für diese Geräusche, deren Schallwellen sich naturgemäß ungehindert im Epipharynx ausbreiten können und in diesem Hoblraum, der ja als.Resonator wirkt, verstärkt werden, giebt es nun drei Wege zur Fortleitung nach der Panke: die Luft, die Tubenwände und den Kathetersehnabel. Die erstere befindet sich, wie oben erwähnt, in starker entgegengesetzter Bewegung, die unter hohem Druek durch eine enge Passage stattfindet, so daß die Schallwellen mehrfach gebrochen, zum Teil vernichtet werden und nur zum geringeren Teile den Abschnitt $b$ erreichen können. Die Tubenwände spielen wohl nur eine sehr geringe Rolle, obwohl gerade in oder an ihnen die Geräusche entstehen; denn erstens handelt es sich in erster Linie um Schleimhaut, also einen festweiohen Körper, der nicht so gut leitet wie ein 
fester Körper, und dann ist die Schleimhantauskleidung der ganzen Tube durch die massierende Wirkung der Luftdusche in Schwingungen versetzt, in denen die betreffenden Schallwellen untergehen müssen. Etwas günstiger ist der dritte Weg, jedoch wohl nur für gröbere und laute Geräusehe, die dnrch Schwingungen größerer Schleimbautfalten und Sekretballen hervorgerufen werden. Denn diese Geräusche - das beweist die häufig fühlbare Vibration des Katheterpavillons - werden durch direkten Kontakt der schwingenden Massen dem Katheter mitgeteilt und so auf den nach der Paukenhöhle gerichteten Luftstrom in dem Instrument übertragen. Auch hier wird eine erhebliche Abschwächung eintreten, da der Schall verschiedene Medien, Luft - Metall - Luft durchdringen mub, und von dem Luftstrom im Katheter nur ein ganz geringer Teil in gerader Richtung in die Pauke eintreten kann.

Aus diesen Erwägungen ergicbt sich, was auch meine Versuche dartun, daß sich der Fortplanzung der Geräusche vom Rachen nach der Pauke starke Hindernisse entgegenstellen. Schwache Geräusche werden daher auf diesem Wege untergehen müssen, und auch laute Geräusche werden nur stark abgeschwächt durch den ins $\mathrm{Ohr}$ eingeführten Auskultationsschlauch wahrgenommen werden können.

Die komplizierteste Bewegung finden wir sicher im $A b$ sehnitte b. Der Luftstrom tritt hier, nachdem er eben den Kathetersehnabel verlassen hat, in ein sich bis zum Isthmus immer mehr verjüngendes Rohr von fast $2 \mathrm{~cm}$ Länge ein. In seiner Verlängerung, mit ihm dureh den engen Isthmus kontinuierlich verbunden, findet sich das komplizierte Raumsystem der knöchernen Tube plus Paukenhöhle plus Adnexe. Die Luft in diesen Räumen steht, wie oben erwähnt, bald nach Beginn der Lufteintreibung unter demselben Drucke wie die den Katheter verlassende Luft. Die Folge davon ist, daß in dem Tubenabsobnitt $b$ der - nehmen wir zunächst an - ganze Luftstrom umkehren mub, um das Ostium pharyngeum zu gewinnen. Da nun der Luftstrom, analog der Mechanik der Blutbewegung in den Gefäßen, in seiner Achse am schnellsten sich fortbewegt, und er mit diesem Teile, vorausgesetzt, daß Tuben- und Katheterachse zusammenfallen, auf kein festes Hindernis stoßen kann, so wird dieser Teil des Luftstroms am längsten seine Vorwärtsbewegung beibehalten. Ich komme später auf diesen Kernstrahl zurück. Die diesem nach außen sich konzentriseh zunächst an- 
schließenden Luftschichten werden dagegen kurz vor dem Isthmus an der Tubenwand und an der unter gleichem Drucke stehenden Luft des knöchernen Tubenabschnitts anprallen und schleifenförmig umkehren; dasselbe, nur noch früher, werden die sich weiter nach außen anschließenden Schichten tun müssen, da sie ja schon auf Luftmengen treffen, die unter gleichem Drucke stehend - naturgemäß der Tubenwandung entlang sich in rïckläufiger Bewegung befinden. Am schnellsten und leichtesten werden die Randpartien umkehren, da sie ja auch die geringste Bewegungsenergie besitzen. Die gesamte Luftmenge gelangt so an dem Kathetersehnabel vorbei in den $A b$ schnitt a.

Diese Luftbewegungenwerden wir, wie erwähnt, dann finden, wenn die Achse der Tube und die verlängerte Katheterschnabelachse zusammenfallen. In praxi wird dies jedoch in der Mebrzahl der Fälle nicht eintreffen, da der Arzt meistens zu einer Stelle des Ostiumrandes mit dem Katheterschnabel Fühlung suchen wird. Hauptsächlich kommt hierbei der obere Winkel des Ostium pharyngeale in Betracht, in zweiter Linie die vordere Wand. Die Grïnde hierfïr sind bekannt genug. Dureh diese Katheterlage wird nun eine von der soeben beschriebenen etwas verschiedene Luftbewegung im Absehnitt $b$ und $c$ verursacht werden. Jedoch handelt es sich nur um eine Modifikation, nicht um eine prinzipielle Änderung. Nehmen wir an, daß der Katheterschnabel dem oberen Winkel anliegt, so liegt es auf der Hand, daß zwischen ihm und der oberen Tubenwand keine Luft pharyngealwärts entweichen kann. Trotzdem werden die mit geringerer Bewegungsenergie ausgestatteten Randzonen des Luftstrables auch in ihren oben gelegenen Teilen frtihzeitig umkehren müssen, zumal ihre Bewegung durch Reibung an der Tubenwand noch weiter verlangsamt wird. Sie müssen daher seitlich umbiegen und medial und lateral vom Kernstrahl und dem Katheterschnabel den Rüekweg zum Pharynx suchen. Auf diese Weise wird der Kernstrahl allmählich die obere Tubenwand gewinnen und im oberen Winkel des Isthmus in die Paukenhöhle eintreten. In der Bewegung der direkt nach unten umbiegenden Luftsohichten tritt naturgemäß gegen die erste Beschreibung keine Änderung ein. Die Bewegungen der Luftschichten im Abschnitt $b$ sind also in beiden Fällen in Form eines Federbusches angeordnet. Im ersten hängen die Federn gleichmäßig nach allen Seiten, im zweiten nur nach einer Seite. 
Die Geräusche, die in diesem Abschnitt b entstehen, werden wohl nur zum geringsten Teile durch schwingende Schleimhautfältchen hervorgerufen werden, da das Tubenlumen durch den starken Luftdruck etwas erweitert, und so etwaige Falten gröBtenteils verstrichen werden. Wir haben es deshalb hier meistens mit Rasselgeräuschen zu tun. Diese werden durch Flüssigkeiten verursacht, die entweder aus der Pauke stammen, oder in der Tube selbst abgesondert, oder artifiziell in die Tube transportiert worden sind. Die je nach Konsistenz und Menge des Exsudates an Eigenart, Frequenz und Stärke versehiedenen Geräusche können sich in dem engen Tubenlumen senkrecht zur Längsacbse naturgemäB leicht ausbreiten. Ihre Schallwellen werden daher sowohl den tympanal- als den pharyngealwärts geriehteten Luft-

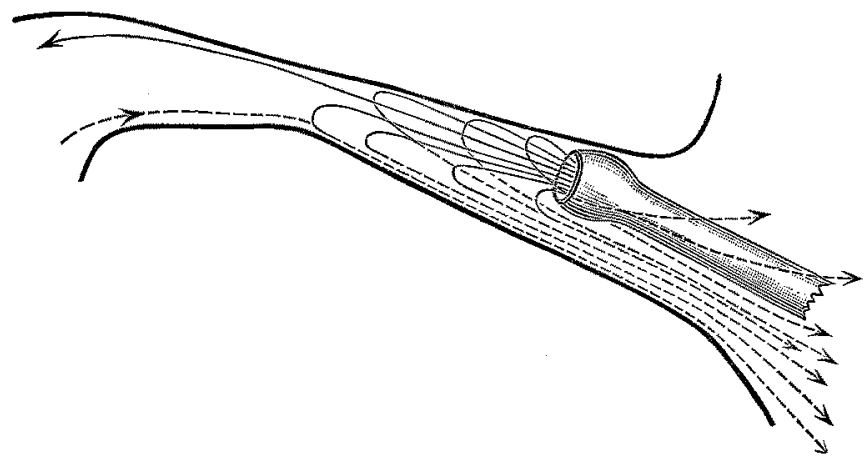

Fig. II.

strom erreichen und so Wege finden, die relativ frei von Luftwirbeln und anderen Leitungshindernissen die Fortpflanzung nach der Pauke und dem Nasenrachenraum ermöglichen. Ebenso werden die Schallwellen der lauten und mittellanten pharyngealen Geräusche, deren Intensität die Erreichung des tympanalwärts fließenden Luftstroms gestattet, sich nach den Mittelohrräumen, abgeschwächt und durch Brechung undeutlicher und versehwommen, fortpflanzen können.

c) Der Abschnitt o der Tube ist m. E. physikalisch nur mit der eigentlichen Pauke zusammen zu betrachten, da das Ostium eigentlich nur nach unten bin durch eine Schwelle gekennzeichnet ist, während sonst der Übergang allmählich erfolgt. Auf Grund zahlreicher klinischer Untersuchungen bin ich nun zu der Überzengung gelangt, daß, auch bei gleichmäßigem Luftstrom, wie wir ihn durch das Lucäsche Doppelgebläse er- 
zielen, ein geringer Luftwechsel in der Pauke stattfindet. Des auch dann habe ich Geräusche beobachten können, die zunal durch Adhäsionen oder konsistentere Flüssigkeitsansamglungen hervorgerufen wurden und sicher nicht in der Tube tstanden. Das beweist auch die Möglichkeit, Dämpfe in dí Xauke einzutreiben. Ich erinnere da besonders an Salpiakdämpfe, die mit vorsichtigem, ganz gleichmäßigem Druck en etrieben werden, um ein Mitreißen von reizenden Flüssigkeiten zu vermeiden.

Diesen geringen Luftweehsel erkläre ich mir folgendermaßen. Am Isthmus kehrt, wie oben erwähnt, die dem Kernstrahl benachbarte Luftzone in Form eines pilzförmigen Kranzes von Schleifen oder eines nach einer Seite umgelegten Federbusches um. Der rỉoklänfige Teil dieser Schleifen bewegt sich an der Tubenwand entlang. Diese umkehrende Luftmenge übt nun eine Saugewirkung auf die im knöehernen Tubenabsohnitt, und zwar im peripheren Teile, lagernde Luft aus, wodureh eine gewisse der Tubenwandung anliegende Menge dieser Luft mitgerissen wird. Besonders wird das in den beiden Winkeln des spaltförmigen Isthmus stattfinden, bzw. im unteren Winkel, wenn der Katheterschnabel der oberen Tubenwand anliegt. Das Defizit an Luft wird ergänzt durch den Eintritt der im obenerwähnten Kernstrahl enthaltenen Luft.

Ich lasse es dahingestellt, welche Kraft als die primäre für diese Wechselwirkung anzusehen ist, ob die Energie des Kernstrahles oder die Saugewirkung des umkehrenden Luftstroms. Das aber steht für mich fest, daß die Bewegung niemals eine absolut kontinuierliche sein kann. Sondern wie der Luftstrahl den Katheterschnabel in wellenähnliehen Vibrationen verläbt, so wird auch diese Bewegung dureh schnell sich folgenden und sich abweehselnden höheren und niederen Druck ausgelöst werden.

Bei meinen Versuchen unter III ist mir der Nachweis gelungen, dab selbst unter relativ günstigen Bedingungen - offener von Luftwirbeln freier Tube und großer Stärke der Geräusche in der Pankenhöhle erzengte Geräusche nicht von der Nase aus hörbar sind. Dafür können nur zwei Gründe maßgebend sein. Bei der Ausbreitung der Schallwellen von Paukengeräuschen wird nur ein verschwindender Bruchteil dieser Wellen auf den schmalen Isthmusspalt entfallen. Diese geringe Schallmenge muß theoretiseh natürlich dureh die Tube in den Nasenrachenraum und schließlich in den Hörschlauch gelangen, jedoch ist ihre 
Streunng so groß und ihre Intensität trotz der Resonanz im Epipharynx so gering, daß in praxi, wie meine Leichenversuche dartun, nichts wahrgenommen werden kann. Dazu kommen am Lebenden als zweiter Faktor die bei Katheterismus tubae in der Ohrtrompete erzengten Luftströmungen und Geräusche. Die ersteren werden, wie ich oben Gelegenheit hatte zu erwähnen, so schwache Schallenergien vernichten, und allein schon das Blasegeränsch wird ausreichen, sie völlig zu verdecken. Gewiß wird der randlänfige Luftstrom aus der Pauke die Fortpflanzung von Paukengeräuschen unterstützen, jedoch ist er einerseits sehr schwach, andererseits mischt er sich dem in heftigen Wirbelbewegungen befindlichen umkehrenden Luftstrom bei, so daß seine begünstigende Wirkung sicher schon im Tubenabschnitt $b$ verloren geht.

\section{Klinische Untersuchungsresultate.}

Wenn ich jetzt zu den praktischen Versuchen und Erfahrungen am Lebenden übergehe, so möchte ich folgendes vorausschicken: Die Anschaungen, die ich in den letzten $\mathrm{Ab}$ schnitten niedergelegt habe, sind lediglich aus Versuchen an Leichen und am herausgenommenen Schläfenbeine gewonnen, sie könnten somit nur auf ganz normale Verhältnisse am Lebenden übertragen werden. Denn mas müßte annehmen, daß Exsudatmengen und Schwellungszustände in Pauke und Tube wesentlich andere physikalische Verhältnisse schaffen müßten. Doch habe ich mich im Laufe meiner praktischen Untersuchungen überzeugt, daß nur verhältnismäßig selten meine Untersuchungsmethode nicht zum Ziele führt. Das traf besonders bei hochgradigen Schwellungszuständen mit relativer und absoluter Stenose der Tube, bei festem, nicht schwingungsfähigem narbigen Tubenversehluß nach chronischen Eiterungen am Ostium tympanicum und ähhnlichen Fällen zu, bei denen überhaupt der Katheterismus tubae nicht oder nur mangelhaft ausführbar ist. Schleimansammlungen am Ostium pharyngeum und im vordersten Tubenabschnitt haben mir wohl im Anfang Schwierigkeiten in den Weg gelegt und manchen Versuch vereitelt oder wenigstens verschleiert. Jedoch habe ich bald gelernt, diese beweglichen Hindernisse, wenn sie nicht durch veränderte Katheterlage zu umgehen waren, durch vorsichtiges, langsames Wegblasen in den meisten Fällen zu beseitigen. Der Luftstrom dringt so nicht in die Pauke ein, und das Bild der Auskultation der Pauke ist 
dann nachber klar. Es empfiehlt sich, bei dieser vorbereitenden Manipulation den Kopf leicht nach hintenuber neigen zu lassen, um jedes Mitreißen von Paukenexsudat zu vermeiden.

Aber auch so bleibt eine Reihe von Fällen, deren Deutung dadurch erschwert ist, daß mehrere ähnliche oder einander deckende und deshalb schwer zu differenzierende Geräusche in $\mathrm{Ohr}$, Tube und Epipharynz entstehen und letztere trotz der eben erwähnten Vorsichtsmaßregeln nicht zu beseitigen sind. Zur Deutung solcher komplizierten Phänomene gehört naturgemäß eine genaue Vergleichung und Abwägung der Stärke, Eigenart und Frequenz der Geräusche, die schon mit geringer Übung erlernt werden kann. Ich lasse ein solehes der Diagnose wohl zugängliches Beispiel hier folgen.

1) Otto Regel, abheilende chronische Eiterung rechts, kleine Perforation, in der Pauke schleimiges Exsudat, das durch Luftdusche wiederholt entfernt warde. Katarrh der oberen Luftwege.

$0 \mathrm{hr}$ : sofort mittelblasiges, sehr nahes fenchtes Rasseln, bei stärkerem Druck mit pfeifendem Blasen in sehr hoher Tonlage vermischt.

Nase: rauhes schlürfendes Blasegerăusch, zeitweilig leises Surren in mittlerer Tonlage, dazwischen spärliche Rasselgeräusche, die bei geringerem Druck meist ganz fehlen, während die Rasselgeränsche aus der Pauke bestehen bleiben.

Wir haben hier Geräusche, die auf katarrhalische Erkrankung des ganzen Tractus auditorius vom Ostium pharyngeum tubae bis zum perforierten Trommelfell hinweisen: Von Ohr und Nase sind Rasselgeräusche hörbar, jene sind jedoch stets vorhanden, frequent, mittelblasig, feucht, diese zäh und spärlich, sie verschwinden zeitweilig, während jene bestehen bleiben. Es findet sich ferner vom Gebörgang aus ein pfeifendes Perforationsgeräusch, während von der Nase aus ein Blasegeräusch von rauhem, schlürfendem Charakter und zeitweilig ein mäßiges Surren in mittlerer Tonlage wahrnehmbar ist. Es ist leicht, diese so klar sich unterscheidenden Einzelgeräusche auseinanderzuhalten und zu lokalisieren. Ähnlich bin ich bei anderen Untersuchungen zum Ziele gekommen.

Aus der großen Reihe meiner kliniseben Untersuchungen habe ich natürlich nur eine eng begrenzte Anzahl zur Mitteilung ausgewählt. Ich habe sie hauptsächlich im Monat April d. J. gesammelt, während ich mir später nur bei besonders markanten Fällen Notizen gemacht habe. Den Hauptwert lege ich auf diejenigen Befunde, die am Lebenden bei gesehlossenem Trommelfell gewonnen sind. Denn gerade in diesen Fällen ist es mir am schönsten gelungen, Unklarheiten zu beseitigen und genauen 
Einblick in gewisse physikalische Vorgänge zu gewinnen. Ich teile diese Versuche in drei Gruppen ein.

a) Geräusche, die nur vom Ohre aus börbar sind.

2) Karl Gunther, abheilende Eiterung, Trommelfell geschlossen, beginnt abzublassen.

Ohr: anfangs rauhes Blasegeräusch mit nahen, mäbig frequenten, klanglosen Rasselgeräuschen. Dann vibrierendes tönendes Surren bei wei cherem Blasen.

N as e: lautes Blasegerărusch, nur im Beginn von schlürfendem Cbarakter. Junge.

3) Subakuter Katarrh, mit Myringitis nach Ausspritzen. ca. 9jăhriger

O h r : Blasen und langsam anfeinander folgende, quappende nahe Rasselgeräusche (zâhes sekret in der Panke).

Nas e: reines Blasegerăusch.

4) Prczy bylski, abheilende Eiterung rechts, Trommelfell geschlossen.

Ohr: zähes nahes Rasseln neben dem Blasegeräusch und Nachrasseln nach Aufhören des Luftstroms.

Nase: nur weiches Blasen.

5) Gu stav Müller, 39 Jahre, Adhäsivprozesse, rechts.

Ohr: Anschlagegeräusch, breites scharfes Blasegeräusch, im Beginn der Lufteintreibung mit feinen knisternden Geräuschen, die nach Aufhören des Luftstroms noch einmal, und zwar stärker, hervortreten.

N a se: nur Blasen ohne Nebengeräusche, nur bei sehr starkem Druck tritt in der Nase starkes Pfeifen auf, gleichzeitig mischt sich anch dem vom Ohre zu auskultierenden Blasegeräusch ein Pfeifen bei.

6) Albert Seifert, Schwerhörigkeit mit Sausen, 63 Jahre. Die Diagnose war von autoritativer Seite auf exsudativen Katarrh gestellt. Trommelfell völlig reizlos.

Ohr: Neben dem Blasegeräusch nahes metallisch klingendes Rasseln von mäßig frequenter, etwas unregelmäßiger Klangfolge. Das Geräusch blieb sich trotz lange fortgesetzter Luftdusche auch bei senkrecht gestellter Tubenachse völlig gleich.

Nas e: dauernd nnr weiches Blasen.

Das Phänomen blieb bei stetig fortschreitender Hörverbesserung in längerer Beobachtung unverändert. Ich stellte die Diagnose auf Adhăsivm prozesse.

7) Auguste Böning, 48 Jahre. (Göttinger Universitätspoliklinik). Adhäsivprozesse, Schwerhörigkeit, Sausen links. Trommelfell reizlos, leicht getrübt.

O h r: Blasegeränsch mit sonorem surrenden Beiklang.

Nase: reines weiches Blasegerăusch.

8) Zickert, 17 Jahre. Deviatio septi, Muschelhypertrophie, Adenoide geringen Grades, exsudativer Katarrb. Links, Exsudatlinie verläuft in einer Wellenlinie und erreicht den Umbo nicht (bereits einige Zeit in Behandlung). Trommelfell deutlich eingezogen.

O br: bei autrechter Kopfhaltung weiches Blasen. Danach ist das Trommelfell ausgebaucht, mäBig injiziert und trübe (chagriniert). Bei stark gesenkter Kopfbaltung treten einige feuchte Rasselgerausche, die von Ohr und Nase gleichmäßig hörbar sind, auf, um bald $\mathrm{zu}$ verschwinden. Dann konnte ich beliebig oft wiederholen: sanftes Blasegeräusch bei leichtem Druck. Ging ich zu stärkerem Druck über, so konnte ich ein deutliches "Tip"-Geräusch bören, das von stärkerem, etwas sonorem Blasegeräusch (feuchte and geschwellte Schleimhaut) gefolgt war.

Nase: wie oben erwähnt, vorübergehend fenchtes nahes Rasselgerăusch. Sonst nur graduell verschiedenes nicht sonores Blasegeräusch, kein "Tip" Geräusch zu hören. (Vgl. anch Versuch 12).

b) Gerâusche die von Ohr und Nase etwa gleich lant hörbar sind.

9) Bernhard S teckner. Nervöse Schwerhörigkeit, beiderseits. Hyperämie des Trommelfells und der oberen Luftwege. 
Rechts: normaler Auskultationsbefund

Links: $0 \mathrm{hr}$ : breites, etwas rauhes Blasen mit einzelnen nahen, zähen Rasselgeräuschen.

Nase: breites, rauhes und lautes Blasen, mit spärlichen, zăhen, ganz deutlichen Rasselgeräuschen. Hier war exsudativer Katarrh neben der Labyrinthaffektion durch einseitige Auskultation diagnostiziert worden, die nasale und aurale Auskultation wies nach, daß das Bekret sich lediglich in der Tube befand.

10) Rudolf Mohr, 56 Jahre, Schwindel infolge Sklerose der Hirnarterien. Auskultationsbefund rechts. Normales Trommelfell.

$\mathrm{O} \mathrm{hr}$ : anfangs reines Blasegerăusch, dem sich nach wenigen Lufteintreibungen deutliche zähe, dann klingende, zuletzt noch grobe brodeinde Rasselgeräusche beimischen. Letztere klangen verschwommen.

Nase: anfangs reines Blasen dann an Stärke zunehmende feuchte Rasselgeräusche, zuletzt sehr lautes Rasseln (bruit de pavillon).

Da am herausgenommenen Katheter nur etwas Schleim klebte, kann es sich nur um Sekretion der Tube handeln, die durch die Manipulation mit dem metallisehen Instrument angeregt war.

12) Zickert, 17 Jabre. Erste Untersuchung, unter 8 ist eine spätere Untersuchung berichtet. Trommelfelle beiderseits gelbrot, stark hervortretender radiărer Gefaßkranz, starke Einziehung.

Rechts: unklares Bild, da neben Rasselgeräuschen von Ohr und Nase gleichzeitig störende lante Geräusche am Ostium pharyngeale auftreten.

Deshalb links: anfangs sanftes Blasen. Zunächst nur von der Nase einige nahe Rasselgeräusche, die bald verschwinden, Trommelfell danach unverändert. Dann ein kräftiger Luftdruck, dabei vom $0 \mathrm{hr}$ : sehr nahes, fast unangnehm lautes, giucksendes Geräusch, kein eigentliches Rasseln. Trommelfell ist danach stark vorgewölbt und trübe, chagriniert und gerötet. Darauf ist sofort bei nächstem Luftdruck von Nase und $0 \mathrm{hr}$ ans feuchtes mittelblasiges sehr frequentes Rasseln zu hören. Nach längerer Lufteintreibung bei gesenkter Kopfhaltung bleiben nur noch sehr spärliche Rasselgerăusche zurück, die dumpf und entfernt vom Ohre, nahe von der Nase gehört werden.

13) A. B., abgelaufener Katarrb, kein Exsudat mehr vorbanden. Trommelfell blaß. Rechts: Anfänglich leises entferntes Blasegeräusch, dann bei stärkerem Druck plötalich starkes Blasen, dasselbe von $\mathrm{Ohr}$ und $\mathrm{Nase}$, hier lanter, hörbar. Die auffallende Erscheinung besteht darin, dab zwei verschiedene Stărken des Blasegerăusches ohne Übergang einander folgen. Der Luftdruck war zum Teil durchaus gleichmäßig, von mittlerer Stärke. Selbst bei kräftigem Druck war kein typisches Anschlagegeränsch zu erzielen.

Weitere positive Resultate habe ich wiederholt bei der Behandlung der Tubenstenose mit Suprarenin (Stammlösung 1:1000) gewonnen. In gleicher Weise wie Politzer bereits Injektionen von Kokain zu diesem Zweeke empfiehlt, wandte ieh dieses weit weniger giftige und die Gewebe viel intensiver zum Abschwellen bringende Präparat an. Ich brachte etwa 3 Tropfen in den Kathetertrichter bei rtiekwärts gebeugtem Kopfe und blies sie mit vorsichtigem Drucke in die Tube ein. Wiederholt fand ich nach einer Dosis und 5-10 Minuten langem Abwarten die Ohrtrompete futr den Luftstrom durchgängig; ; bei einem Falle mufte ich zweimal drei Tropfen injizieren, in zwei Fällen kam ich nur mit der Bougie zum Ziele. Es ist mir so gelungen, die nicht immer sehonende und daher nicht unbedenkliehe Anwendung der Bougie in einem großen Prozentsatz zu vermeiden. 
Über eine vorübergehende nachträgliche Verschlimmerung der Symptome des Tubenversehlusses, die man auf reaktive Schwellung nach dem Ablauf der Suprareninwirkung bätte beziehen können, wurde nicht geklagt. Es liegt auf der Hand, daß alte Stenosen, die auf Hynertrophie des Bindegewebes beruhen, dieser Maßnahme unzugänglich sind, doch wird auch bier dureh vorherige Suprarenininjektion die Einführung der Bongie erleichtert werden.

Ich konnte bei diesen Fällen, stets dann, wenn überhaupt ein, wenn auch enger; Weg für den Luftstrom vorhanden oder erst durch die Suprareninwirkung geschaffen worden war, anfangs frequentes, dann spärliches feuchtes Rasseln, zuletzt, wenn das Medikament verteilt war, nur ein dem Blasegeräusch beigemischtes Schlürfen hören. Diese Geränsche waren deutlich von der Nase, je nach der Weite der Tube mehr oder weniger gedämpft vom Ohre aus hörbar; sie ermöglichten mir vielfach eine exakte Kontrolle, ob das Suprarenin richtig appliziert war.

Dasjenige Geräusch dieser Klasse, das am allerhäufigsten beim Gelingen des Katheterismus zur Beobachtung gelangt, nur dann nicht, wenn es durch Nebengeräusche verdeckt wird, ist das Blasegeräusch in seinen verschiedenen Abstufungen vom weichen Blasen bis zum scharfen ,Chi-Geräuseh" (Schwartze l.c.) Die Art und der Ort seiner Entstehung steht wohl immer noch zur Diskussion. Man hat sich das Blasegeräuseh durch das Einströmen der Luft in die Pauke entstehend gedacht, man hat seinen Ursprung in der Tube und schließlich am Katheterschnabel gesucht. Für seinen verschiedenen Klangcharakter hat man die Beschaffenheit der Sehleimhant in Pauke oder. Tube, die Weite der letzteren und schließlich die Weite des Katheterschnabels verantwortlich gemacht. Auf Grund meiner Untersuchungen bin ich zu folgender Ansicht gelangt:

Die Pauke ist als Entstehungsort auszuschließen, da dort entstehende Geräusche nicht von der Nase aus gehört werden könnten, und gerade das unkomplizierte Blasegeräuch von $\mathrm{Ohr}$ und Nase stets gleichen Klangeharakter hat. Einseitig wahrnehmbare Nebenklänge oder Nebengeräusche sind nach meinen Untersuchungen stets außerhalb des Ursprungsortes des Blasegeräusches entstanden, also entweder in der Pauke, wenn sie nur vom Ohre, oder am Tubenostium, wenn sie nur oder stark überwiegend von der Nase aus hörbar sind. Andererseits ist die Luftströmung in der Pauke sehr gering, sie wirkt hauptsächlich als Resonator, und, wie Uffenorde nachgewiesen hat, 
das Blasegeräusch ist vom Vorhandener sein einPauke völlig unabhängig.

Eine Einwirkung von seiten des Katheterschnabels habe ich dadurch ausgeschlossen, daß ioh für sämtliche Versuche stets gleich weite Katheter verwendet habe.

Das Blasegeräusch entsteht vielmehr nur zum allergeringsten Teile durch die Vibrationen des den Katheterschnabel verlassenden Luftstroms, zum größten Teile dureh Reibung an den Tubenwänden (Uffen orde 1. e.), und zwar hauptsäehlich im Abschnitt b und an der Grenze von $b$ und a. Denn der Charakter des unkomplizierten Blasegeräusches ist stets derselbe, ob vom $\mathrm{Ohr}$ oder von der Nase gehört, nur entspreehend stärker oder sehwäeher. Ist der Ursprungsort so durch diese Untersuchungen festgestellt, so ist die Frage nach der Ursache des verschiedenen Klang-

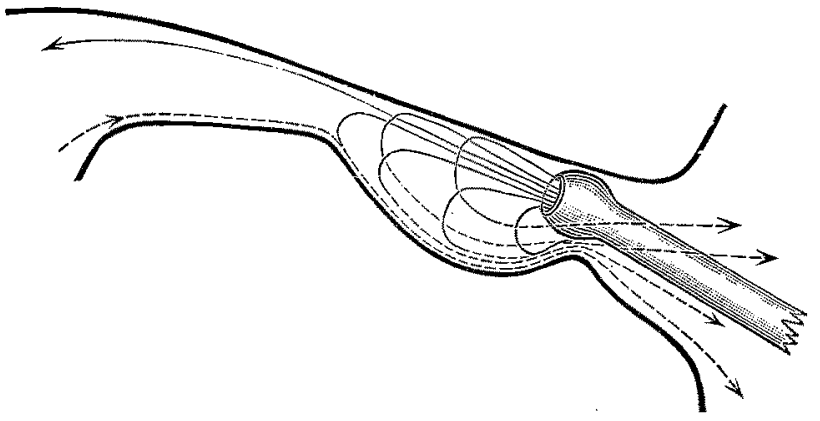

Fig. III.

charakters bedentend schwieriger zu beantworten. Ich habe dazu die Entstehung des "Chi"Lantes im Munde beobachtet, und zwar alle Übergänge vom weichen Hauchlaut an bis zum scharfen "Chi". Man bringt ihn dadureh hervor, daß man den Zungenrïcken allmählich immer mehr gegen den harten Gaumen hebt, bis beim "Chi" nur ein sohmaler Spalt übrigbleibt. Gleichzeitig wird das Gaumensegel gegen die hintere Rachenwand gehoben. Man kann das Spiel der Tubengaumenmuskeln selbst durch Ohrgeräusche kontrollieren. Die Folge davon ist, daß sich in der hinteren Mund- und der Rachenhöhle ein Hohlraum bildet, in dem sich der Expirationsdruck staut, um sohließlich durch den schmalen Spalt zwisehen Zungenrüeken und harten Gaumen zu entweichen.

Analoge Verhältnisse liegen m. E. beim Katheterismus vor. Hier stant sich die den Katheterschnabel verlassende Luft zunächst unter hohem Druck im knorpelig-membranösen Tuben- 
absehnitt $b$, um dann gedrängt von der nachfolgenden Luft in Schleifenform umzukehren und das Ostium zu gewinnen. Wir haben hier also dasselbe Bestreben eines Luftstrahles, der auf ein Hindernis - sei es Luft oder ein fester Körper - stößt, sich pilzförmig nach den Seiten auszubreiten, wie wir es beobachten können, wenn wir Zigarrendampf in dickem Strahl in die Luft oder gar gegen eine Wand blasen. Der Luftstrom in der Tube muB ja allerdings wegen der allseitig entgegenstehenden Wandungen gänzlich kehrt machen, aber doch wird sich dieses zur Strahlachse senkrechte Auseinanderstreben des gestauten Luftstroms so weit geltend machen können, als die individuelle Dehnbarkeit der knorpelig-membranösen Tube besonders nach außen and unten hin es zuläßt. Die Tubenwandungen werden daher im Bereiche der Stromumkehr - also im Abschnitt b mehr oder weniger auseinandergedrängt, also sackartig erweitert, und die Luft muß dann etwa in der Höhe des Kathetersehnabels durch den engeren vorderen Tubenabschnitt a in einheitlichem Strome unter langsam abnehmendem Drucke nach dem Nasenrachenraum entweichen. Diese Verengerung kann vielleicht durch Verziehung der Schleimhaut, auf die ich schon oben hindeutete, noch stärker in Form einer Schwelle hervorspringen. Die Verschärfung des Blasegeräusches richtet sich natülich graduell nach dem zwisehen Tubenabsehnitt $b$ und a bestehenden Querschnittsunterschiede. Die Übereinstimmung mit der Entstehung des „Chi"-Lautes im Munde ist demnach vollständig.

c) Im Tubenabschnitt a entstehende Gerăusche.

Bei Beobachtung dieser Geräusche, die ja auch bei auskulkultatorisch normalen Paukenräumen auferordentlich häufig auftreten, habe ich stets den oben ausgesprochenen, durch Leichenversuche gewonnenen Satz hestätigt gefunden, dab laute Geräusche von der Nase natürlich sehr laut, vom Ohre erheblich abgeschwächt, mittellaute Geräusche hier nur als Beiklang, leise Geräusche dagegen nur von der Nase aus hörbar sind. Die Fortpflanzungsmöglichkeit tympanalwärts ist bei tönenden Geräuschen am größten, bei klanglosen Rasselgeräuschen am geringsten. Eine Rolle spielt auch der Umstand, dab die schwingenden Massen zuweilen den Katheter beriihren. Ich möchte mieh auf die Mitteilung von nur zwei Beispielen beschränken.

14) Grauert, Tubenkatarrh, links.

O hr: scharfes Blasegerăusch, zeitweilig bei starkem Druck von helltönendem Charakter. 
Nase: sehr scharfes Blasen, zeitweilig bei starkem Druck mit Pfeifen vermischt.

15) Lucie Wiege, Residuen nach Katarrh, rechts.

$0 \mathrm{~h} \mathbf{r}$ : durchweg reines Blasegeräusch.

$\mathrm{N}$ a se: lantes Blasen, gegen Ende des Versuches feuchtes mittelblasiges klangloses Rasseln, anfangs leise, dann bis mittelstark, das vom Ohre nicht hörbar ist. Es findet sich Blut am Katheter.

Dieselbe Erfahrung habe ich vor kurzem noch einmal gemacht.

d) Ergebnisse bei bestehender Trommelfellperforation.

Anch beim Bericht über meine zahlreichen Untersuchungen bei Patienten dieser Klasse kann ich mich kurz fassen, da ich stets dieselben Resultate gewonnen habe. Ich habe niemals Perforationsgeräusche, sei es Rasseln oder Pfeifen selbst von sehr intensiver Stärke, von der Nase aus wahrnehmen können. Es ist das ja auch daraus verständlich, daß bei freier Verbindung der Pauke mit dem Gehörgang ein viel größerer Bruchteil der Luft durch den Tsthmus hindurchtritt und dieser Luftstrom wohl die Fortpflanzung nach dem Gehörgang, aber nieht rückwärts nach dem Rachen unterstützt. Außerdem findet in diesen Fällen kein Rückströmen von Luft aus der Pauke in den Tubenkanal statt, und in den meisten Fällen wenigstens wird das Perforationsgeräusch am Trommelfell, also etwas weiter vom Isthmus entfernt erzeugt. Aber auch wenn das Pfeifen - z. B. bei sehr großen Perforationen - durch schwingende Schleimhautfältchen oder Sekrettröpfchen in der Pauke erzeugt wird, habe ich das Phänomen nur vom Ohr aus wahrnehmen können. Immerhin gebe ich die Möglichkeit zu, daß lant pfeifende Geräusche vielleicht dann von der Nase aus gehört werden können, wenn sie bei großen Trommelfellperforationen am Isthmus tubae selbst oder in seiner unmittelbaren Nachbarschaft entstehen. Außer dem bereits unter 1 mitgeteilten führe ich nur zwei Beispiele an.

16) Przybylski, Akute Mittelohreiterung. Enge Trommelfellperforation. Unter 4 ist eine spätere Untersuchung mitgeteilt.

$0 \mathrm{hr}$ : auf mittleren Druck prasselndes Gerăusch von zerplatzenden Blasen mit Pfeifen vermischt.

Nase: nur mäßig scharfes Blasen.

17) Otto Plavius, abheilende chronische Eiterung. Große zentrale Perforation, sehr geringe schleimige Sekretion. Mucosa geschwellt.

Ohr: hohes Pfeifen mit einzelnen Rasselgeräuschen. gerăusch.

N a se: schlürfendes, später leicht sonores (tiefer Toncharakter) Blase-

e) Ergebnisse bei ausgeschaltete n Mittelohrräumen.

Schon Uffenorde hat darauf hingewiesen, daß in Fällen von Totalaufmeißlung, wenn nur die Tuba Eustachii dureh eine schwingungsfähige Membran verschlossen ist, sowohl das Blasegeräusch als auch sonstige in der Tube entstehende Geräusche 
ebenso deutlich gehört werden, als seien sie im Mittelohr entstanden. Man mub annehmen, daß diese Geräusche anch von der Nase ans hörbar sind. Und das trifft anch zu, bis auf das Anschlagegeräusch, das ỉch außer dem zu erwähnenden Falle noch einmal in gleicher Weise beobachten konnte. Das weist darauf hin, daß dieses "Tip"-Geräusch, wie bei intaktem Mittelohr im Trommelfell, so hier in der am Ostium tympanioum tubae ausgespannten schwingungsfähigen Narbenmembran und nicht in der Tube entsteht, also als Ausbanchungsgeräusch aufzufassen ist. Dann aber zeigt diese Beobachtung auch, dab für die Fortleitung der Paukengeräusche nach dem Rachen Pauke und knöcherner Tubenabschnitt physikalisch als ein einheitliches Ganze betrachtet werden müssen.

18) Hermann Elste, Totalaufmeißlung. Ohr trocken, Tube narbig verschlossen.

$0 \mathrm{hr}$ : sehr deutliches weiches Blasegeräusch, zeitweilig gedämpftes Rasseln und Pfeifen.

$\mathrm{Na}$ se: dasselbe, nur deutlich, laut und nahe.

18) Friederike Grey, Totalaufmeißlung beiderseits, beide Ohren trocken, Tuben geschlossen.

Rechts: Ohr: weiches Blasegeräusch mit spärlichen "nahen" feuchten Rasselgeräuschen.

Na s e: lantes weiches Blasegerăhusch, daneben gleich stark wie vom Ohre spärliches feuchtes Rasseln.

Links: Von $\mathrm{Ohr}$ und $\mathrm{N}$ ase weiches Blasegerăusch.

19) A. Z. "Spontane Totalaufmeißlung“, ausgeheiltes Cholesteatom. Pauke trociken. Tube durch Membran geschlossen.

O hr: weiches nahes Blasegeränsch, eingeleitet durch deutliches Anschlagegeräusch.

Nas e: weiches Blasen, auch bei kurzen kräftigen Lufteintreibungen nur rein ein weiches Anblasen.

Nachdem ich die drei hauptsächlichsten Leitsätze bereits am Schluß der Leichenversuche zusammengestellt habe, möchte ich nur noch kurz die praktischen Schlußfolgerungen anführen, die ich aus meinen Untersuchungen an $\mathrm{Pa}$ tienten gewonnen habe.

Geräusehe, die durch Adhäsionen in der Pauke hervorgerufen werden, sind aaturgemäb nur vom Ohre auskultierbar. Ich konnte sie einmal (Fall 6) nur dadurch von fenchten Exsudatgeränschen unterscheiden, dab ich duroh die nasale Auskultation das Ausbleiben von sicher zu erwartenden Tubengeräuschen feststellte. In einem zweiten Falle (7) stellte ich die Diagnose dadurch, dab ich feststellte, daß das Geräusch (sonorer, surrender Beiklang) nicht vom Rachen oder von der Tube her fortgeleitet sein konnte, sondern in der Paukenhöhle entstand.

Bei entzündlichen Prozessen läßt ein solcher nur vom Ohre 
wahrnehmbarer Beiklang auf starke Durehfeuchtung und Schwellung der Paukensehleimhaut sebließen $(2,8)$.

Ist ein Exsudat nur von geringer Menge, so kann es in der Paukenhöhle verteilt (2) oder auch bei stark geneigtem Kopf durch die Tube entfernt werden (8); zuweilen treten erst bei Anwendung dieses Hilfsmittels Rasselgeränsche anf und ermöglichen bei undurchsichtigem Trommelfell so die Diagnose (8). Bei zähem Exsudat gelang in unseren Fällen die Entfernung nicht $(3,4)$.

Das Anschlagegeräusch entsteht nach unseren Ergebnissen mit Sicherheit in der Pauke und nicht in der Tube. Es kommt in dem Augenblicke zu stande, wenn das Trommelfell oder eine seine Stelle vertretende Membran den Grad seiner höchsten Spannung bei der Ausbanchung nach dem Gehörgang zu erreicht (IV, 5, 19). Das tritt meistens fast gleichzeitig mit dem Eindringen der Luft in die Panke ein, kann jedoch auch etwas später stattfinden, z. B. bei schlaffem Trommelfell (8). Wird das Trommelfell durch Exsudat beschwert, so findet sich das Anschlagegeräusch erst nach Beseitigung der Flüssigkeit (8).

Das Abheben der Tubenwände scheint sich nicht durch das "Tip"-Geräusch, sondern nur durch übergangslose, graduell verschiedene Stärke des Blasegeräusches zu doknmentieren (13).

Wir können mit Hilfe der nasalen Auskultation die Entfernung von Sekret aus der Paukenhöhle durch die Tube genau kontrollieren $(8,12)$, ebenso die Verteilung von Flïssigkeiten, die zu therapeutischen Zwecken in die Tube, eventuell in das Mittelohr eingeblasen werden. Mit der nasalen Auskultation läßt sich feststellen, daß der überwiegende Teil der Rasselgeräusche nicht in der Pauke, sondern in der Tube beim Abfließen des Exsudats entsteht $(8,9,10,12,18)$.

Rasselgeräusche, die von Anfang an von Ohr und Nase gleich laut und frequent und gleichartig gehört werden, sind auf Sekret zu beziehen, das in der Tube lagert $(9,18)$. Es kann auch erst durch den Reiz des Katheterismus die Tube zur Sekretion angeregt werden (10).

Das Blasegeräusch (bruit de pluie) entsteht im knorpeligen Tubenabschnitt, sein Charakter (weich, scharf, "Chi"-Geräuseh) ist abhängig von der Form des Tubenabschnitts.

Ein rauhes Blasegeräusch, das, von der Nase gehört, einen schlürfenden Charakter hat, wird durch Schwellung und starke Befeuchtung der Tubenschleimhaut bedingt. 